\title{
Lackfehler besser erkennen in neuem Lichttunnel
}

\section{Zur besseren Kontrolle der}

Oberflächenqualität wurde ein neuer

Lichttunnel entwickelt. Das Lichtkonzept

und die ergonomische Umgebung

ermöglichen ein exaktes und

hochkonzentriertes Sehen. Dadurch lassen

sich Unregelmäßigkeiten im Lack konstant

mit hoher Genauigkeit erkennen.

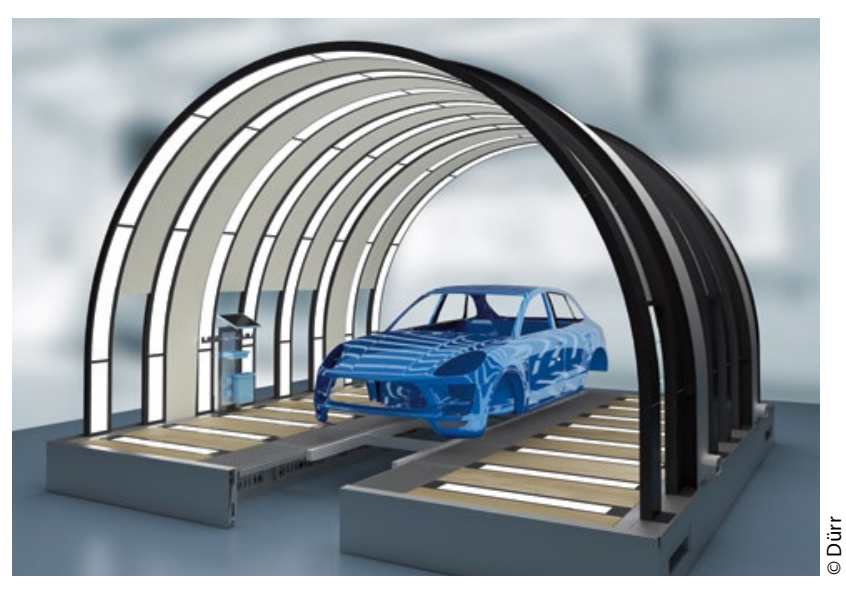

Der neue Lichttunnel bietet visuell und ergonomisch optimale Voraussetzungen, um die Qualität von Lackoberflächen zu kontrollieren.
Bevor Karossen aus der Lackiererei in die Endmontage übergehen, wird die Lackstruktur auf kleine Fehler, wie Einschlüsse von Staub oder Härchen, überprüft. Für diese diffizile Tätigkeit in der Qualitätskontrolle schafft der Lichttunnel EcoReflect von Dürr visuell und ergonomisch gute Voraussetzungen.

Um Abweichungen zu erkennen, ist ein Reflexbild mit hohen, aber nicht zu reizintensiven Kontrasten erforderlich. Die Anzahl der Kontrastlinien im Blickfeld orientiert sich bei dem Lichttunnel an einer passenden Reizfrequenz.

Anders als bei herkömmlichen Systemen werden hier vertikale statt horizontale Kontrastlinien erzeugt. Dies sorgt für ein ergonomischeres und entspannteres Arbeiten, da die Arbeiter den Kopf nicht auf und ab bewegen müssen, um dem Lauf der Karossen folgen zu können.

Blendfreies Sehen ist eine weitere wichtige Voraussetzung, um Oberflächenfehler zu erkennen und zugleich Ermüdungserscheinungen während der Arbeit zu minimieren. Entsprechende Prismen an den Leuchten sorgen dafür, dass möglichst viel Licht auf die Karosserie strahlt und die Arbeiter nicht geblendet werden. Unterstützend dabei wirkt das Strahlungsprinzip der asymmetrischen Beleuchtung.
Auch das Leuchtenkonzept mit LED-Modulen hat Dürr weiterentwickelt. Neu ist, dass sowohl hohe Kontraste im Reflexbild erzeugt werden als auch Gebiete mit homogener Ausleuchtung vorhanden sind. Die verwendete LED-Technologie benötigt nur die Hälfte der Energie herkömmlicher Leuchtstoffröhren. Auch gegenüber standardmäßigen LED-Ausführungen ist EcoReflect sparsamer, da die Bogenform des Lichttunnels besonders viel Licht auf die Karosserie bringt.
Das System kann als Einzelplatz gebaut werden oder als Linie. Es ist für komplexe Fehlertypen erweiter- und individualisierbar hinsichtlich Lichtfarben und Materialien. //

\section{Kontakt}

Dürr Systems AG, Bietigheim-Bissingen Tel. 07142780

corpcom@durr.com, www.durr.com

\section{Nachfolger für Unternehmen im Bereich Oberflächenbehandlung/Pulverbeschichtung/ Nasslackierung gesucht.}

Aus Altersgründen soll das Unternehmen komplett mit Grundstück, Gebäuden und Maschinenpark veräußert werden.

Standort NRW Köln-Düren

Die Zuschriften bitte an folgende Chiffrenummer: 082564 